\title{
Inhibition of Noncanonical Murine Double Minute 2 Homolog Abrogates Ocular Inflammation through NF- $\kappa$ B Suppression
}

\author{
Yan Fan, Wei Zhang, Aiguo Ni, Biraj Mahato, and Sai H. Chavala
}

From the Department of Pharmacology and Neuroscience, University of North Texas Health Science Center, Fort Worth, Texas

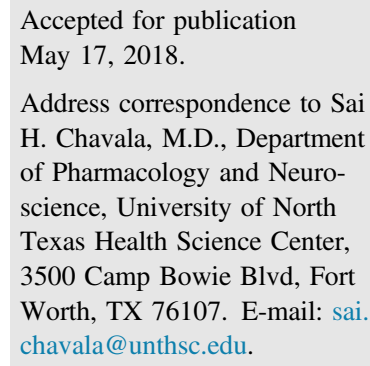

\begin{abstract}
Uveitis is estimated to account for $10 \%$ of all cases of blindness in the United States, including 30,000 new cases of legal blindness each year. Intraocular and oral corticosteroids are the effective mainstay treatment, but they carry the risk of serious long-term ocular and systemic morbidity. New noncorticosteroid therapies with a favorable side effect profile are necessary for the treatment of chronic uveitis, given the paucity of existing treatment choices. We have previously demonstrated that Nutlin-3, a small-molecule inhibitor of murine double minute 2 (MDM2) homolog, suppresses pathologic retinal angiogenesis through a p53-dependent mechanism, but the noncanonical p53-independent functions have not been adequately elucidated. Herein, we demonstrate an unanticipated function of MDM2 inhibition, where Nutlin-3 potently abrogates lipopolysaccharide-induced ocular inflammation. Furthermore, we identified a mechanism by which transcription and translation of NF- $\kappa \mathrm{B}$ is mediated by MDM2, independent of p53, in ocular inflammation. Small-molecule MDM2 inhibition is a novel noncorticosteroid strategy for inhibiting ocular inflammation, which may potentially benefit patients with chronic uveitis. (Am J Pathol 2018, 188: 2087-2096; https://doi.org/10.1016/j.ajpath.2018.05.017)
\end{abstract}

Uveitis is a leading cause of irreversible blindness ${ }^{1}$ and represents a collection of inflammatory eye diseases, which mainly affect the iris, ciliary body, and choroid. ${ }^{2}$ Corticosteroids are effective in treating ocular inflammation and preventing blindness, but they often become intolerable during the chronic treatment of uveitis. ${ }^{3-7}$ Systemic corticosteroids are effective in reducing ocular inflammation, 3 but have the risk of serious adverse effects, such as glucose intolerance, hypertension, and osteoporosis., ${ }^{4,7}$ Intraocular steroids have reduced systemic adverse effects, but they can result in vision loss by the development of cataract and steroid-induced glaucoma., ${ }^{5,6}$ Thus, the development of new noncorticosteroid therapies with a favorable adverse effect profile is warranted to suppress ocular inflammation for the treatment of chronic uveitis.

Murine double minute 2 (MDM2) is classically known to degrade tumor suppressor p53 through its E3 ubiquitin ligase activity, $^{8}$ and small-molecule inhibitors of MDM2 are currently being evaluated as an anticancer therapy in clinical trials for their antiproliferative and proapoptotic effects. ${ }^{9,10}$ MDM2 has now been reported to have non-p53-dependent cellular functions, such as NF- $\mathrm{NB}$ induction both in vitro and in vivo, but its role in nonischemic inflammation is not known. ${ }^{11-13}$ In our previous study, we demonstrated that inhibition of MDM2 with small molecule effectively suppresses ocular angiogenesis through a p53-dependent mechanism. ${ }^{14}$ In the current study, we sought to investigate the noncanonical p53-indendepent function of MDM2 inhibition in ocular inflammation using lipopolysaccharide (LPS)-induced ocular inflammation. Herein, we demonstrated that inhibition of

Supported by the Nancy Lee and Perry R. Bass Endowment, the Foundation Fighting Blindness, and National Eye Institute awards EY021171 and EY025667 (S.H.C.).

Disclosures: S.H.C. filed patent application 61/386,808, "MDM2 inhibitors for treatment of ocular conditions," on September 27, 2010. S.H.C. is a founder of Serrata LLC, a start-up company that plans to commercialize novel treatments for ocular diseases. 
MDM2 potently abrogates ocular inflammation through $\mathrm{NF}-\kappa \mathrm{B}$ suppression, independent of p53. Small-molecule MDM2 inhibition is a novel noncorticosteroid strategy for inhibiting ocular inflammation in chronic uveitis.

\section{Materials and Methods}

\section{Chemicals and Reagents}

Ethyl alcohol, dimethyl sulfoxide, LPS (from Escherichia coli 0111:B4), actinomycin D (ACTD), and cycloheximide (CHX) were purchased from Sigma (St. Louis, MO). $( \pm)$ Nutlin-3 was purchased from Cayman Chemical (Ann Arbor, MI).

\section{Animal and Tissue}

C57/BL6 mice (wild type) and $p 53^{+/-}$mice were purchased from The Jackson Laboratory (Bar Harbor, ME). Three-week-old $p 53^{-/-}$mice underwent intravitreal (IVt) injection with $1 \mu \mathrm{L}$ of $10 \mu \mathrm{mol} / \mathrm{L}$ Nutlin-3 in $10 \%$ ethanol $^{14}$ and/or i.p. injection with $100 \mu \mathrm{L}$ of $40 \mathrm{mg} / \mathrm{kg}$ Nutlin-3 in $30 \%$ dimethyl sulfoxide. ${ }^{11}$ Eyes with vehicle injected were included as control. After 16 hours, mice were intraperitoneally injected with $200 \mathrm{mg}$ LPS, and a second dose of Nutlin-3 was intraperitoneally injected at the same time. Choroids and retinas were isolated 7 hours after LPS injection. The time point for isolating choroids and retinas was determined on the basis of our initial time course experiments, in which the peak cytokine mRNA expression occurred 7 hours after injection (Supplemental Figure S1). Three independent experiments were performed, and each experiment had three eyes per group. In total, $36 p 53^{-1-}$ mice were used for these experiments. To study the effect of systemic Nutlin-3 in local LPS-induced ocular inflammation, mice were intraperitoneally injected with $100 \mu \mathrm{L}$ of $40 \mathrm{mg} / \mathrm{kg}$ Nutlin-3. After 16 hours, $1 \mu \mathrm{L}$ of $250 \mathrm{ng}$ LPS was intravitreally injected, and a second dose of Nutlin-3 was intraperitoneally injected at the same time. Vehicle-injected eyes were included as control. Eyes were enucleated 7 hours after LPS injection. Three independent experiments were performed, and each experiment had three eyes per group. In total, 12 wild-type mice and 12 $p 53^{-1-}$ mice were used in this study. To detect the effect of Nutlin-3 on retinal cell death, adult wild-type mice were intravitreally injected with a supratherapeutic dose of 250 $\mu \mathrm{mol} / \mathrm{L}$ Nutlin-3 once a week for 4 weeks and vehicle was injected as a control. Noninjected mice were also included as control. The retinas were analyzed using hematoxylin and eosin staining, spectral domain-optical coherence tomography (OCT), and electroretinography (ERG) 4 weeks after the final injection. Three independent experiments were performed, and each experiment had three eyes per group. In total, 15 wild-type mice were used in this study.

\section{Cells and Cell Culture}

ARPE-19 cells were purchased from ATCC (Manassas, VA) and were seeded at a density of $5 \times 10^{4}$ cells per well in a 6-well plate. Cells were infected with $N F-\kappa B$ reporter lentivirus encoding firefly luciferase under control of a minimal cytomegalovirus promoter and tandem repeats of the $N F-\kappa B$ transcriptional response element (Cellomics Technology, Halethorpe, MD). Two days later, the cells were cultured in medium with $2 \mu \mathrm{g} / \mathrm{mL}$ puromycin and selected for 10 days. After selection, ARPE-19-NF- $\kappa B$-Luciferase reporter cell line (ARPE-19-NF- $K B$-luc) cells were maintained in Dulbecco's modified Eagle's medium Nutrient Mixture F-12 medium (Thermo Fisher Scientific, Waltham, MA), supplemented with $10 \%$ fetal bovine serum, $50 \mathrm{U} / \mathrm{mL}$ penicillin, and $50 \mu \mathrm{g} / \mathrm{mL}$ streptomycin in a $37^{\circ} \mathrm{C}, 5 \% \mathrm{CO}_{2}$ incubator. Before treatment, cells were starved in serum-free medium for 24 hours. To detect the role of MDM2 inhibition in LPS-induced inflammation in ARPE-19 cells, cells were treated with indicated amount of Nutlin-3 7 hours before $10 \mathrm{ng} / \mathrm{mLLPS}$ was added. To inhibit gene transcription and protein translation, cells were pretreated with $10 \mu \mathrm{mol} / \mathrm{L}$ ACTD or $10 \mu \mathrm{g} / \mathrm{mL} \mathrm{CHX} \mathrm{for} 1$ hour, respectively, followed by Nutlin-3 and LPS treatment. Cells were harvested for analysis 4 hours after LPS treatment.

\section{Transfection}

ARPE-19-NF- $\kappa B$-luc cells were plated in $60-\mathrm{mm}$ plates at a density of $1 \times 10^{6}$ cells/plate or in 24-well plates at a density of $1 \times 10^{5}$ cells/well. Then, cells were transfected with 80 $\mathrm{nmol} / \mathrm{L}$ nontargeting control siRNA or $p 53$-specific siRNA (Santa Cruz Biotechnology, Dallas, TX) using Promega Fugene HD transfection kit (Promega, Madison, WI), according to the manufacturer's instructions. ARPE-19 cells were plated in 48 -well plates at a density of $5 \times 10^{4}$ cells/well. Cells were transfected with $100 \mathrm{ng} / \mu \mathrm{L}$ RelA Prom plasmid (Active Motif, Carlsbad, CA) using Promega Fugene HD transfection kit (Promega), according to the manufacturer's instructions. After transfection for 16 hours, cells were refilled with normal culture medium. After another 48 hours, cells were first treated with Nutlin-3 in the indicated concentrations for 7 hours, followed by $10 \mathrm{ng} / \mathrm{mL}$ LPS for 4 hours.

\section{Western Blot Analysis}

Cells were washed twice with ice-cold phosphate-buffered saline and lysed in radioimmunoprecipitation assay buffer (Thermo Fisher Scientific), with $1 \times$ protease and phosphatase inhibitor cocktail (Thermo Fisher Scientific) on ice for 20 minutes for whole-cell lysate preparation. Nuclear extracts were isolated using a nuclear/cytosol fractionation kit (BioVision, Milpitas, CA), according to the manufacturer's instructions. Protein concentration was determined using a Pierce BCA Protein Assay kit (Thermo Fisher Scientific), and the optical density at $562 \mathrm{~nm}$ was taken using Biotek Synergy 2 Microplate Reader (BioTek Instruments 
Inc., Winooski, VT). Cell lysates were electrophoretically separated using the Nupage Protein Gel System (Thermo Fisher Scientific). Western blot was analyzed with appropriate primary antibodies: anti-NF- $\kappa \mathrm{B}$ (Cell Signaling Technology, Danvers, MA), anti-p53 (Santa Cruz Biotechnology), anti-histone H3 (Abcam, Cambridge, UK), and anti- $\beta$-actin (Sigma), followed by Luminata Cresendo Western HRP Substrate (Millipore, Burlington, MA). Images were taken using Alpha Innotech FluorChem HD2 imaging system (Alpha Innotech, San Leandro, CA).

\section{Luciferase Activity Assay}

Cells were washed twice with ice-cold phosphate-buffered saline and refilled with $100 \mathrm{~mL} 1 \times$ lysis buffer (Promega), then incubated at room temperature for 5 minutes. The clear supernatant was collected by centrifugation at maximum speed for 20 seconds. The same volume of the supernatants was used for the subsequent luciferase reporter assay. The luciferase activity was quantitated using a Biotek Synergy 2 Microplate Reader.

\section{RNA Isolation and Quantitative PCR}

Total RNA was isolated using Trizol Reagent (Invitrogen) and a Direct-zol RNA MiniPrep kit (Zymo Research, Irvine, $\mathrm{CA}$ ), according to the manufacturer's instructions. RNA concentration was measured using Nanodrop 2000 (Thermo Fisher Scientific). cDNA was synthesized using a High Capacity cDNA Reverse Transcription kit (Applied Biosystems, Foster City, CA). Quantitative PCR was performed using Fast SYBR Green Master Mix (Applied Biosystems) with the following primers: human tumor necrosis factor- $\alpha$ (TNF- $\alpha$ ), 5'-CCCAGGGACCTCTCTCTAATCA-3' (forward) and 5'-GCTTGAGGGTTTGCTACAACATG- $3^{\prime}$ (reverse); human IL-6, 5'-GTAGCCGCCCCACACAGA-3' (forward) and 50'-CATGTCTCCTTTCTCAGGGCTG-3' (reverse); human monocyte chemotactic protein-1 (MCP-1), 5'-ACTCTCGCCTCCAGCATGAA-3' (forward) and 5'TTGATTGCATCTGGCTGAGC- $3^{\prime}$ (reverse); mouse Tnf- $\alpha$, $5^{\prime}$-CCACCACGCTCTTCTGTCTAC-3' (forward) and 5'-AGGGTCTGGGCCATAGAACT-3' (reverse); mouse Il-6, $5^{\prime}$-CTCTGGGAAATCGTGGAAAT-3' (forward) and $5^{\prime}$ CCAGTTTGGTAGCATCCATC- $3^{\prime}$ (reverse); mouse Mcp-1, $5^{\prime}$-CCTGCTGTTCACAGTTGCC-3' (forward) and $5^{\prime}$ ATTGGGATCATCTTGCTGGT-3' (reverse); and human NFкB-p65, 5'-CCCCACGAGCTTGTAGGAAAG-3' (forward) and $5^{\prime}$-CCAGGTTCTGGAAACTGTGGAT- $3^{\prime}$ (reverse). The program for quantitative $\mathrm{PCR}$ is $95^{\circ} \mathrm{C}$ for 10 minutes, followed by 40 cycles of $95^{\circ} \mathrm{C}$ for 15 seconds and $60^{\circ} \mathrm{C}$ for 1 minute, and 1 cycle of $95^{\circ} \mathrm{C}$ for 15 seconds, $60^{\circ} \mathrm{C}$ for 1 minute, and $95^{\circ} \mathrm{C}$ for 15 seconds.

\section{MTT Assay}

Cells were added with MTT $(5 \mathrm{mg} / \mathrm{mL})$ directly to the culture medium to the final concentration of $1 \mathrm{mg} / \mathrm{mL}$ and incubated at $37^{\circ} \mathrm{C}$ for 4 hours. The medium was removed, and the purple crystal precipitates were dissolved in $200 \mu \mathrm{L}$ dimethyl sulfoxide. The solvent was aliquoted into the wells of a 96-well plate, and the OD was determined using a Biotek Synergy 2 Microplate Reader at a test wavelength of $490 \mathrm{~nm}$ and a reference wavelength of $650 \mathrm{~nm}$ and then used to represent the relative cell proliferation.

\section{Cell Death Assay}

After treatment, the viability of ARPE-19 cells was analyzed using a Cell Death Detection ELISA kit (Roche, Basel, Switzerland), according to the manufacturer's instructions.
A

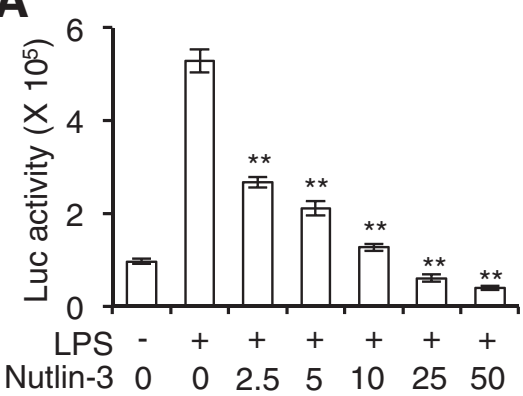

C

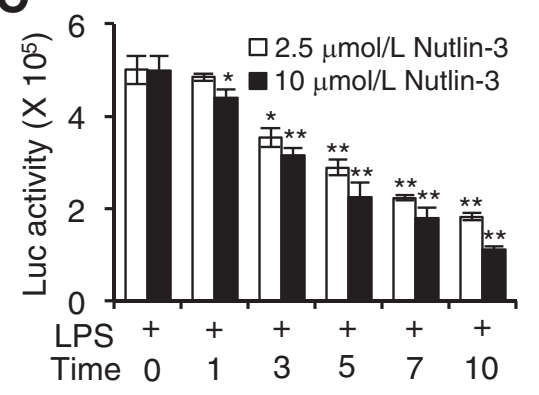

B
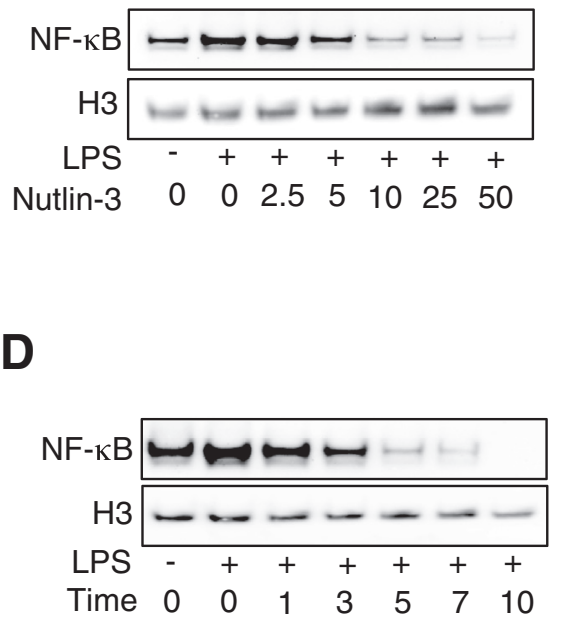

Figure 1 Attenuation of LPS-induced NF-KB activation by MDM2 inhibition in ARPE-19 cells is dose and time dependent. ARPE-19-NF- $K B$-luc (Luc) cells were treated with an indicated concentration of Nutlin-3 ( $\mu \mathrm{mol} / \mathrm{L} ; \mathbf{A}$ and $\mathbf{B}$ ) or for indicated time durations (hours; $\mathbf{C}$ and $\mathbf{D}$ ). Cell lysates were used for detecting luciferase activity ( $\mathrm{A}$ and $\mathbf{C}$ ); nuclear extracts were processed for Western blot analysis to detect NF- $\kappa \mathrm{B}$, and $\mathrm{H} 3$ was included as the loading control (B and D). Data are expressed as means $\pm S D$ of three independent experiments $(\mathbf{A}$ and $\mathbf{C}) .{ }^{*} P<0.05,{ }^{*} P P 0.01$ compared with control. 
Histology and Quantitative Thickness Measurement of Retinal Layers

Eyes were harvested and fixed in neutral-buffered $4 \%$ paraformaldehyde. After paraffin embedding, sagittal sections across the optic nerve were prepared. Sections ( $5 \mu \mathrm{m}$ thick) were cut at five different depths, followed by hematoxylin and eosin staining for morphologic observation of the retinal layers. The average number of infiltrated inflammatory cells within the anterior and posterior segments in three sections from five depths of each eye was manually quantified on microscopic images. Images were taken using a $10 \times$ objective lens on an ACCU-SCOPE EX1310 microscope (Accu Scope Inc., Commack, NY). The thicknesses of various retinal layers were measured using CaptaVision software version 3.6.7 (Accu Scope Inc.). Three measurements from three equivalent regions across the inner nuclear layer, the outer nuclear layer, or the whole retina were obtained in triplicate serial sections from five depths for each eye. Nine eyes for each group were measured and statistically analyzed.

\section{Spectral Domain-OCT}

Spectral domain-OCT scans were performed using the Reveal OCT2 Imaging System (Phoenix Research Laboratories, Pleasanton, CA) using a mouse objective lens provided by the manufacturer. Cross-sectional images, centered on the optic nerve as the main landmark, were obtained from the retina. Retinal thickness was measured at six positions along the cross-sectional image of the retina (average distance of $300 \mathrm{~mm}$ from the optic nerve head) and averaged for each eye. In the vast majority of cases, three positions on each side of the optic nerve head were chosen. The confocal image (wavelength, 750 to $930 \mathrm{~nm}$ ) of the fundus recorded by the OCT system was used for registration.

\section{Electroretinography}

After a minimum of 12-hour dark adaptation, mice were anesthetized by i.p. injection of ketamine/xylazine cocktail $(85 / 14 \mathrm{mg} / \mathrm{kg})$. Animal preparation was performed under a dim red light $(<50$ lux $)$. ERG analyses were performed using a Micron IV Imaging System Ganzfeld ERG (Phoenix Research Laboratories). For the assessment of scotopic response, a stimulus intensity of $-1,0,1,3 \mathrm{Log}\left(\mathrm{cd} . \mathrm{sec} / \mathrm{m}^{2}\right)$ was presented to the dark-adapted dilated eye. The amplitude of the scotopic a-wave was then measured from the prestimulus baseline to the a-wave trough. The amplitude of the $b$-wave was then measured from the trough of the a-wave to the crest of the b-wave. A total of 5 to 10 repeated flashes and measurements were averaged to produce the
A

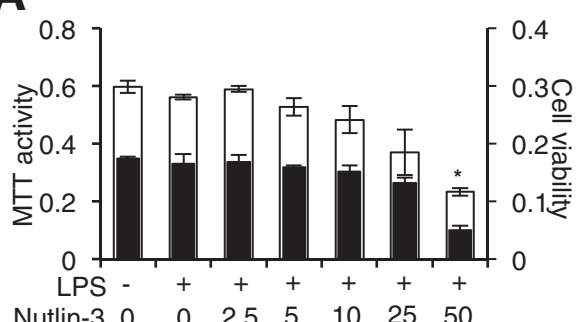

Nutlin-3 $0 \quad 0 \quad 2.5 \quad 5 \quad 10 \quad 25 \quad 50$

C

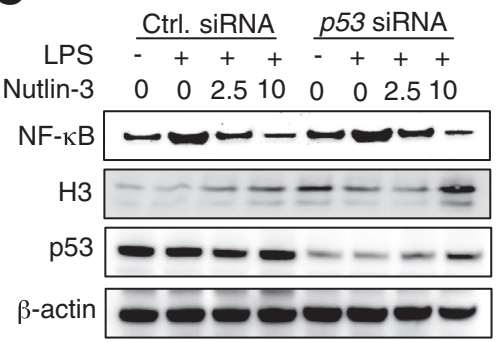

B

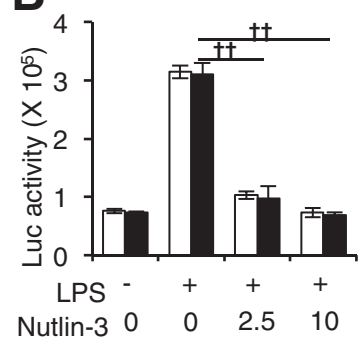

D

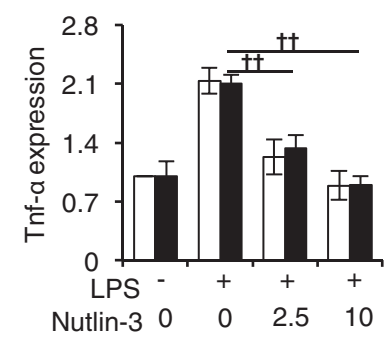

E

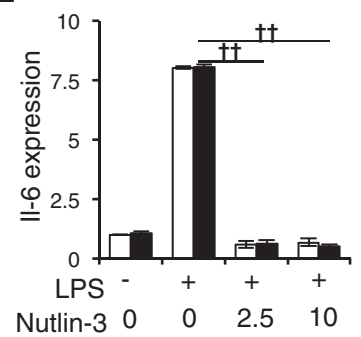

$\mathbf{F}$

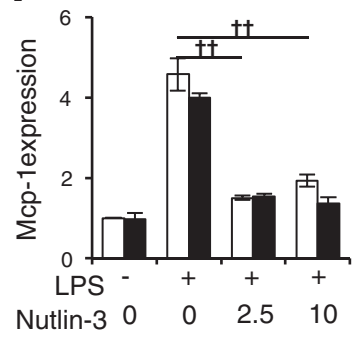

G

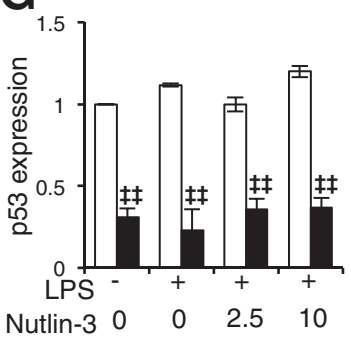

Figure 2 MDM2 inhibition attenuates LPSinduced NF- $K B$ activation in ARPE-19 cells. A: ARPE19 cells were treated with indicated concentration of Nutlin-3 $(\mu \mathrm{mol} / \mathrm{L})$, followed by LPS. Cell lysates were used for detecting MTT activity (open bars) and cell viability (closed bars). B: ARPE-19-NF- $K B-$ luc (Luc) cells were transfected with control (Ctrl.) siRNA (white bars) or $p 53$ siRNA (black bars), and treated with Nutlin-3 and LPS. Cell lysates were used for detecting luciferase activity. C: Nuclear extracts were processed for Western blot analysis to detect NF- $\kappa B$, and $\mathrm{H} 3$ was included as a loading control. Whole-cell lysates were used to detect p53, and $\beta$-actin was used as a loading control. D-G: Total RNA was subjected to quantitative PCR for analysis of Tnf- $\alpha$ (D), Il-6 (E), Mcp-1 (F), and p53 expression (G). Data are expressed as means \pm SD of three independent experiments $(\mathbf{A}, \mathbf{B}$, and $\mathbf{D}-\mathbf{G})$. ${ }^{*} P<0.05$ compared with control; ${ }^{\dagger \dagger} P<0.01$ compared with cells treated with LPS only; ${ }_{\ddagger \ddagger} P<0.01$ compared with cells treated with control SiRNA. 
A

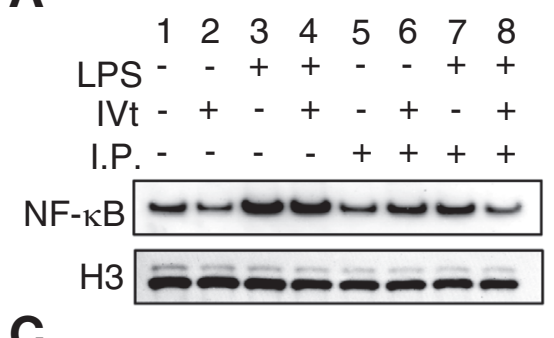

C

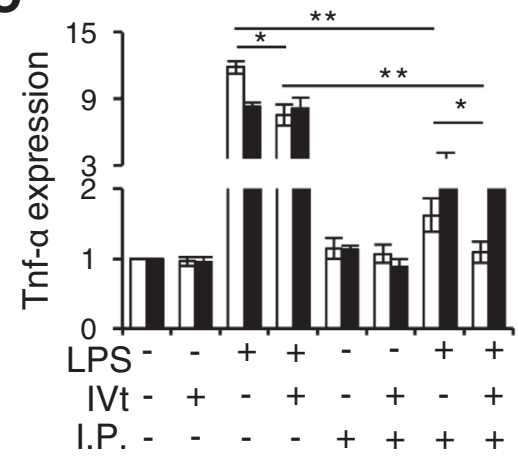

B
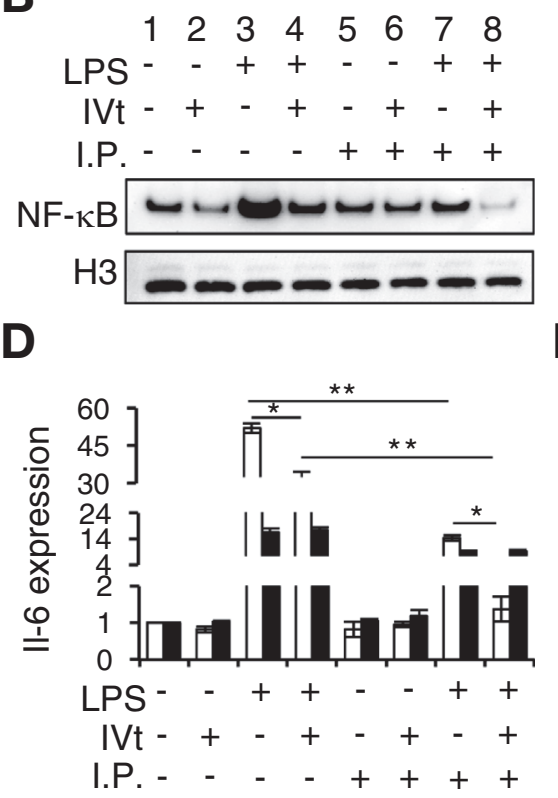

E

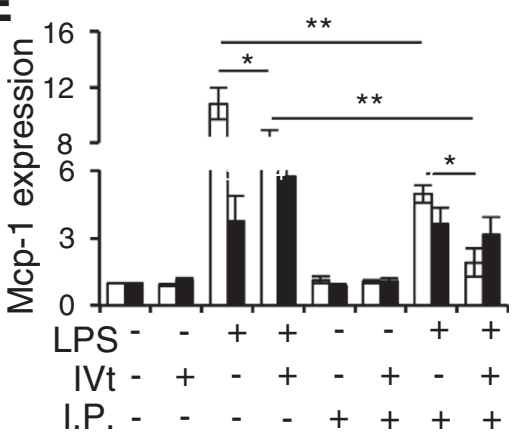

Figure 3 Mdm2 inhibition attenuates LPS-induced NF- $\kappa B$ activation in wild-type mice. Wild-type mice were intraperitoneally (IP) or intravitreally (IVt) injected with Nutlin-3, followed by i.p. LPS. A and B: Nuclear extracts of mouse retina (A) and choroid (B) were processed for Western blot analysis to detect NF- $\mathrm{KB}$, and $\mathrm{H} 3$ was included as a loading control. C-E: Total RNA was isolated from choroid (white bars) and retina (black bars) and used for quantitative PCR analysis of Tnf- $\alpha$ (C), Il-6 (D), and Mcp-1 (E) expression; glyceraldehyde-3-phosphate dehydrogenase was included as a loading control. Data are expressed as means \pm SD of three independent experiments

(C-E). ${ }^{*} P<0.05,{ }^{* *} P<0.01$.

final waveform. At the beginning of the day, the response of wild-type C57B6 mice (aged $>21$ days, $n \geq 2$ ) was recorded and quantified to ensure proper device calibration.

\section{Statistical Analysis}

For each test, three independent experiments were performed. For each independent experiment, the number of mice and the number of eyes are specified in the methods. Data were analyzed using one-way analysis of variance and were presented as means $\pm \mathrm{SD}$ of three independent experiments. $P<0.05$ was considered statistically significant.

\section{Results}

\section{Attenuation of LPS-Induced NF- $\kappa B$ Activation in ARPE-19 Cells by MDM2 Inhibition Is p53 Independent}

Retinal pigment epithelium (RPE) cells play an essential role in photoreceptor maintenance, and in modulating the ocular immune response. ${ }^{15}$ To investigate whether MDM2 inhibition eliminates LPS-induced RPE inflammation, it was tested whether the proinflammatory pathway NF- $\kappa \mathrm{B}$ was activated by LPS and if this could be reduced by MDM2 inhibition. An ARPE-19-NF- $\kappa B$-luc in which luciferase activity served as an indicator for $N F-\kappa B$ activation was generated. Nutlin-3, at various concentrations, was added to the cells, followed by LPS treatment. The data demonstrated that $N F-\kappa B$ was significantly activated by LPS by more than fivefold. LPS-induced $N F-\kappa B$ activation was significantly reduced by $2.5 \mu \mathrm{mol} / \mathrm{L}$ Nutlin-3, and it was reduced nearly to the baseline with $10 \mu \mathrm{mol} / \mathrm{L}$ and higher concentrations of Nutlin-3 (Figure 1A). Western blot analysis of NF- $\kappa \mathrm{B}$ expression from the nuclear extract, a marker for NF- $\kappa B$ activation, revealed a similar result. Five micromoles of Nutlin-3 was sufficient to inhibit NF- $\kappa \mathrm{B}$ expression to baseline (Figure 1B). The time-dependent effect of Nutlin-3 on LPS-induced NF- $\kappa \mathrm{B}$ activation was tested in ARPE-19-NF- $\kappa B$-luc. Significant inhibition of LPS-induced $N F-\kappa B$ activation was observed with 10 $\mu \mathrm{mol} / \mathrm{L}$ of Nutlin-3 after 3 hours of treatment. After 5 hours, significant inhibition was observed in both 2.5 and $10 \mu \mathrm{mol} /$ L Nutlin-3-treated cells (Figure 1C). Western blot analysis revealed a similar result (Figure 1D). MDM2 inhibition is known to increase p53, which leads to inhibition of cell proliferation and induction of cell apoptosis. ${ }^{8}$ To rule out the possibility that NF- $\kappa \mathrm{B}$ suppression is secondary to inhibition of RPE proliferation or induction of cell death, ARPE19 cells were treated with increasing concentrations of Nutlin-3 and no statistically significant inhibition of proliferation or induction of cell death was found at therapeutic concentrations. However, cell viability inhibition was observed with $50 \mu \mathrm{mol} / \mathrm{L}$ of Nutlin-3 (Figure 2A). To determine whether the reduction in NF- $\kappa \mathrm{B}$ activity is $p 53$ independent, reporter cells were transfected with a $p 53$ siRNA, which resulted in $>80 \%$ p53 knockdown (Figure 2, 
A

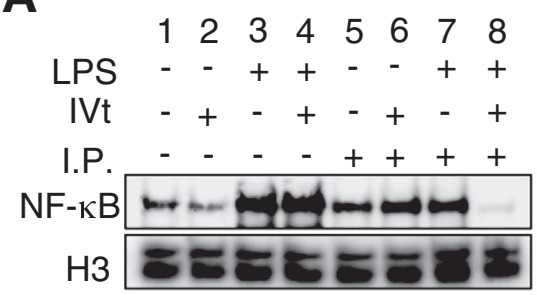

C

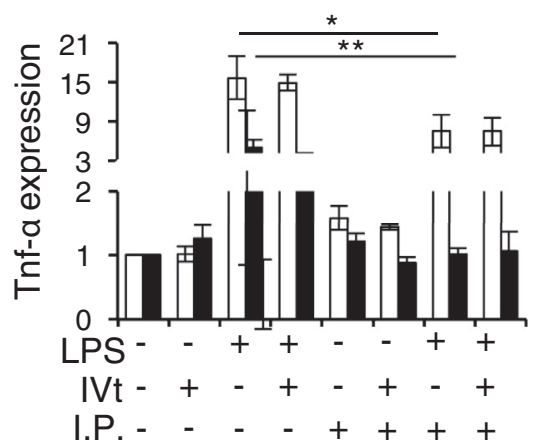

B

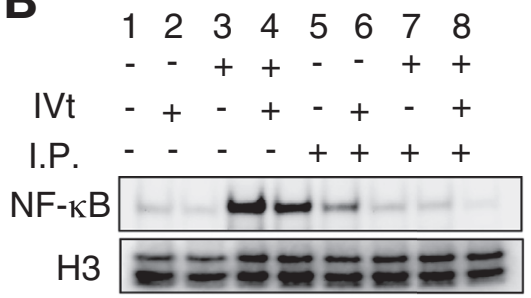

D

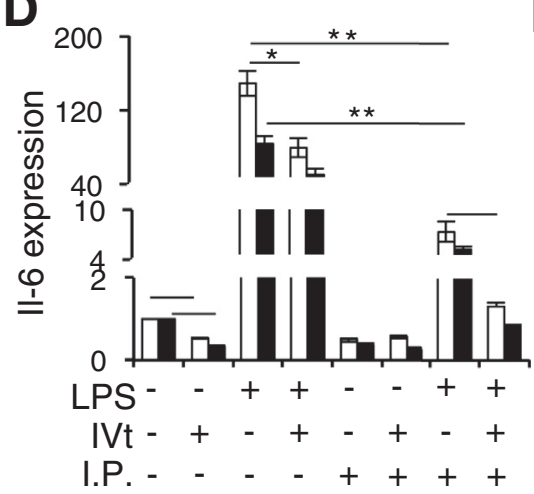

$\mathbf{E}$

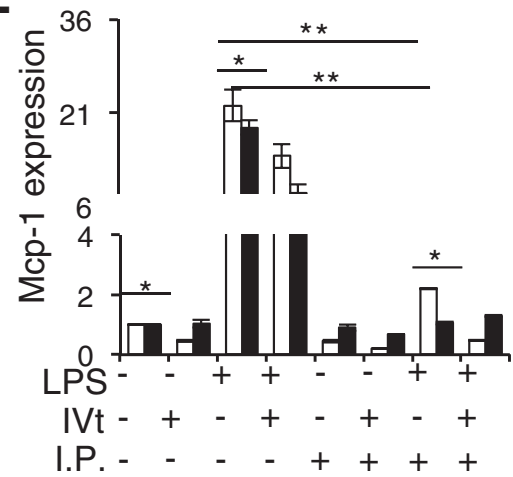

Figure 4 Mdm2 inhibition attenuates LPS-induced NF- $\mathrm{KB}$ activation in $\mathrm{p} 53^{-/-}$mice. The $\mathrm{p} 53^{-/-}$mice were intraperitoneally (IP) or intravitreally (IVt) injected with Nutlin-3, followed by i.p. LPS. A and B: Nuclear extracts of mouse retina (A) and choroid (B) were processed for Western blot analysis to detect NF- $\mathrm{KB}$, and $\mathrm{H} 3$ was included as loading control. C-E: Total RNA was isolated from choroid (white bars) and retina (black bars) and used for quantitative PCR analysis of Tnf- $\alpha$ (C), Il-6 (D), and Mcp-1 (E) expression; glyceraldehyde-3-phosphate dehydrogenase was included as a loading control. Data are expressed as means \pm SD of three independent experiments $(\mathbf{C}-\mathbf{E}) .{ }^{*} P<0.05,{ }^{*}{ }^{*} P<0.01$.

C and G). Cells were then pretreated with Nutlin-3, followed by LPS induction. Inhibition of LPS-induced luciferase $-N F-\kappa B$ activation by Nutlin-3 was observed in both nontargeting control siRNA and p53 siRNA-transfected cells (Figure 2B). To further confirm NF- $\kappa \mathrm{B}$ activation, the nuclear NF- $\kappa \mathrm{B}$ protein level was measured. LPSinduced NF- $\kappa \mathrm{B}$ translocation was inhibited in Nutlin-3 pretreated ARPE-19 cells to near baseline in both the nontargeting control siRNA- and the p53 siRNA-transfected cells (Figure 2C). Next, the levels of downstream targets of NF- $\kappa \mathrm{B}$ were studied using q-PCR. The mRNA levels of Tnf- $\alpha$ (Figure 2D), Il-6 (Figure 2E), and Mcp-1 (Figure 2F) were induced by LPS alone by approximately twofold, eightfold, and fivefold, respectively, compared with control. Pretreatment of Nutlin-3, before LPS induction, resulted in the reduction of the mRNA level of all three cytokines close to baseline in both the nontargeting control siRNA and the p53 siRNA-transfected cells. These data suggest that Nutlin-3 inhibits LPSinduced NF- $\mathrm{BB}$ activation and its downstream cytokine targets in ARPE-19 cells in a p53-independent manner.

\section{Attenuation of LPS-Induced NF- $\kappa B$ Activation by Mdm2 Inhibition in the Wild-Type Mice}

To investigate the in vivo role of Mdm2 inhibition in ocular inflammation, the effect of Nutlin-3 was studied in the LPSinduced uveitis mouse model. Nutlin-3 was administered by i.p. or IVt injection in wild-type mice before i.p. LPS injection. Western blot analysis was performed using nuclear extracts from either retina (Figure $3 \mathrm{~A}$ ) or choroid (Figure 3B), respectively. Increased expression of NF- $\kappa \mathrm{B}$ after LPS injection was observed in both retina and choroid (Figure 3, A and B). IVt injection of Nutlin-3 before LPS treatment resulted in reduced NF- $\kappa \mathrm{B}$ in the choroid more than in the retina (Figure 3, A and B). Remarkably, i.p. Nutlin-3 reduced LPS-induced NF- $\kappa$ B activation in both retina and choroid, with choroid inhibited almost to baseline (Figure 3, A and B). Next, the downstream targets of NF- $\kappa$ B were examined in LPS-induced ocular inflammation. The mRNA levels of Tnf- $\alpha$ (Figure 3C), Il-6 (Figure 3D), and Mcp-1 (Figure 3E) were induced by LPS approximately 13-, 53-, and 11-fold, respectively, compared with control. IVt injection of Nutlin-3 before i.p. LPS injection inhibited the mRNA level of all three cytokines in the choroid. Greater reduction in the mRNA level of all three cytokines was observed by i.p. injection of Nutlin-3 compared with IVt injection in the choroid (Figure 3, C-E). Strikingly, i.p. and IVt Nutlin-3 resulted in a synergistic effect with a robust reduction in the mRNA level of Tnf- $\alpha$, Il- 6 , and Mcp-1, to near baseline in the choroid (Figure 3, C-E).

\section{Attenuation of LPS-Induced NF- $\kappa$ B Activation by Mdm2 Inhibition in $p 53^{-/-}$Mice}

To further investigate the p53-independent role of Mdm2 inhibition in ocular inflammation, in vivo studies were performed in which Nutlin-3 was administered by i.p. or IVt 
A
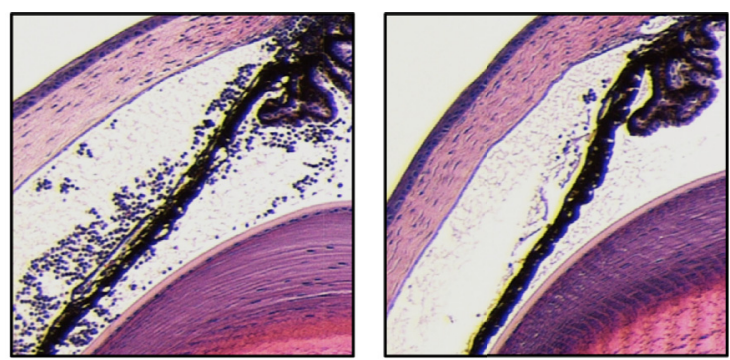

B

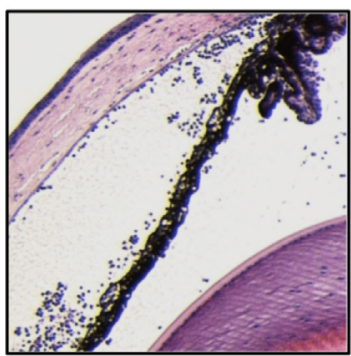

LPS

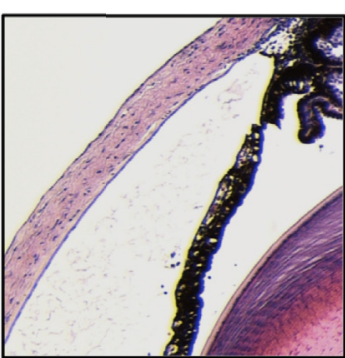

Nutlin-3 + LPS

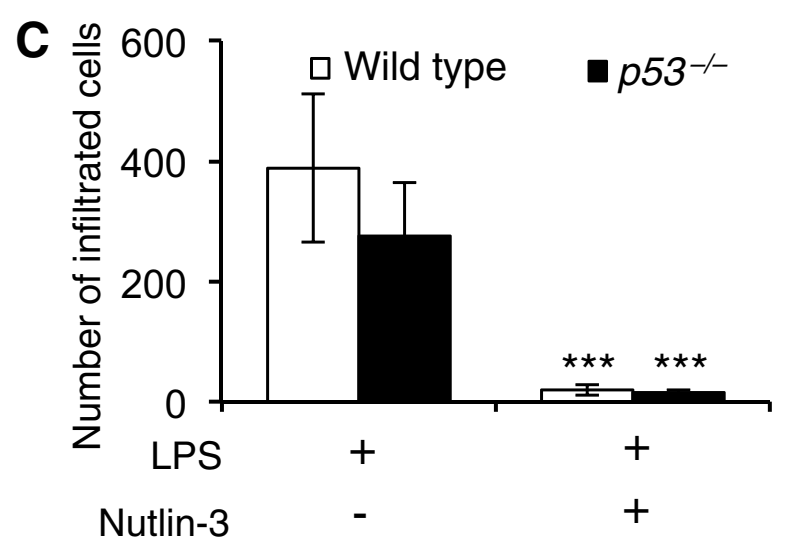

Figure 5 The effect of systemic Mdm2 inhibition in local LPS-induced inflammatory cell infiltration. A and B: Adult wild-type (A) and $p 53^{-/-}$(B) mice were intraperitoneally injected with Nutlin-3, followed by intravitreal LPS, and whole eyes were subjected to hematoxylin and eosin staining. C: Infiltrated inflammatory cells in both anterior and posterior segment of the eye were quantified. Data are expressed as means \pm SD of three independent experiments $(\mathbf{C}) .{ }^{* *} P<0.001$ compared with eyes with only LPS injection. Original magnification, $\times 20(\mathbf{A}$ and $\mathbf{B})$.

injection in $p 53^{-1-}$ mice before i.p. LPS injection. Western blot analysis was performed using nuclear extracts from either retina (Figure 4A) or choroid (Figure 4B), respectively. Increased expression of NF- $\mathrm{B}$ a after LPS injection was observed in both retina and choroid (Figure 4, A and B). IVt injection of Nutlin-3 before LPS treatment resulted in reduced NF- $\kappa \mathrm{B}$ in the choroid more than in the retina (Figure 4, A and B). The i.p. Nutlin-3 reduced LPS-induced NF- $\kappa \mathrm{B}$ activation in both retina and choroid, with choroid inhibited almost to baseline (Figure 4, A and B). Next, the downstream targets of NF- $\kappa \mathrm{B}$ were examined in the LPS-induced ocular inflammation, and similar results to wild-type mice were found (Figure 3, C-E). The mRNA levels of Tnf- $\alpha$ (Figure 4C), Il-6 (Figure 4D), and Mcp-1
(Figure 4E) were induced by LPS approximately 15-, 130-, and 21-fold, respectively, compared with control. IVt injection of Nutlin-3 before i.p. LPS injection inhibited the mRNA level of Il- 6 and Mcp-1 but not Tnf- $\alpha$ in the choroid. Greater reduction in the mRNA level of all three cytokines was observed by i.p. injection of Nutlin-3 compared with IVt injection in retina and choroid (Figure 4, C-E). The i.p. and IVt Nutlin-3 resulted in a synergistic effect, with a robust reduction in the mRNA level of all three cytokines to near baseline in the choroid (Figure 4, C-E).

\section{Systemic Mdm2 Inhibition Is Effective in Inhibiting} LPS-Induced Inflammatory Cell Infiltration in Anterior and Posterior Segments of the Eye

Although the data suggest that systemic LPS-induced ocular inflammation is reduced by Nutlin-3 delivered locally and systemically, it remains unclear if local LPS-induced ocular inflammation can be suppressed by systemic Nutlin-3. Experiments were performed in which i.p. Nutlin-3 injection was followed by IVt LPS injection. Substantial inflammatory cell infiltration was found into the anterior and posterior segments of the eye with IVt LPS in both wild-type and $p 53^{-l-}$ mice (Figure 5, A and B). With i.p. Nutlin-3 before IVt LPS, a dramatic reduction in inflammatory cell infiltration was observed in both anterior and posterior segments (Figure 5, A and B). In the controls, no inflammatory cell infiltration was observed in both vehicle- and Nulin-3-injected eyes (Supplemental Figure S2). Statistical quantification revealed that i.p. Nutlin-3 demonstrated an extremely significant reduction of LPS-induced inflammatory cell infiltration in both wild-type and $p 53^{-1-}$ mice, but no significant difference was observed between LPS and LPS/Nutlin-3-injected eyes of wild-type and $p 53^{-1-}$ mice (Figure $5 \mathrm{C}$ ). Taken together, these results suggest that systemic $\mathrm{Mdm} 2$ inhibition is effective in inhibiting local LPS-induced ocular inflammation independent of p53.

\section{Supratherapeutic Nutlin-3 Does Not Appear to Cause Retinal Cell Death}

To rule out the possibility that Nutlin-3 suppression of ocular inflammation is a result of retinal cell death, the potential adverse effects of intraocular administration of Nutlin-3 were investigated. Wild-type mice were injected with a supratherapeutic dose of Nutlin-3 $(250 \mu \mathrm{mol} / \mathrm{L})$ by IVt injection once weekly for 4 weeks. OCT, hematoxylin and eosin, and ERG (Figure 6) analyses were performed 4 weeks after the final injection. OCT imaging and hematoxylin and eosin staining showed intact laminated retina cell layers with similar thickness in Nutlin-3-injected eyes compared with vehicle-injected control (Figure 6, A-C). ERG analysis demonstrated no statistically significant difference in a- or b-wave amplitudes for Nutlin-3-injected eyes compared with vehicle-injected control (Figure 6, D and E). 
A

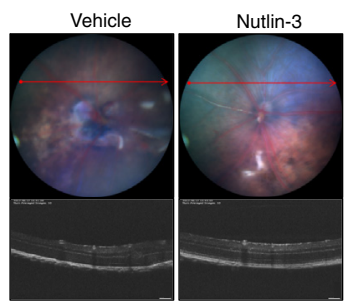

D

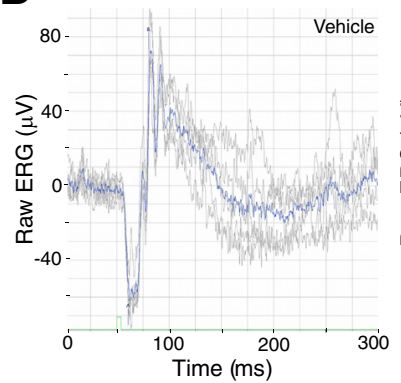

B

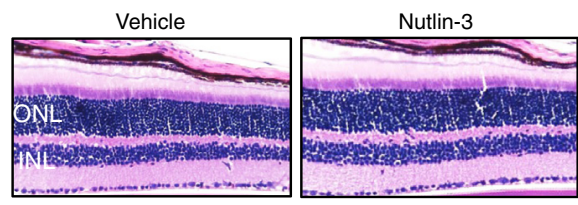

$E$
C

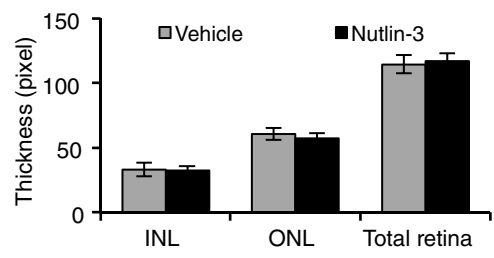

Figure 6 Supratherapeutic dose of Nutlin-3 does not cause retinal cell death. Adult wild-type mice were intravitreally injected with $250 \mu$ mol/L Nutlin-3 once a week for 4 weeks. A-E: Optical coherence tomography (OCT; A), hematoxylin and eosin (B and C), and electroretinographic (ERG; D and E) analyses were performed. A: The red arrow lines indicate the OCT scan locations. B: Quantitative thickness measurements of the inner nuclear layer (INL), the outer nuclear layer (ONL), and total retinal layers were statistically analyzed. E: Quantification and statistical analysis for a- and b-waves was performed. Scale bar $=$ $100 \mu \mathrm{m}(\mathbf{A})$.

\section{LPS-Induced Transcription and Translation of NF- $\kappa \mathrm{B}$ Are Suppressed by MDM2 Inhibition}

Although the data suggest that ocular inflammation suppression is p53 independent, it remains unclear how MDM2 inhibition leads to NF- $\mathrm{BB}$ activity suppression. To investigate the underlying mechanism, it was first tested if Nutlin-3 affects $N F-\kappa B$ transcription or mRNA stability. The transcription in ARPE-19 was inhibited with ACTD, followed by Nutlin-3 and LPS treatment. Without ACTD, the LPSinduced NF- $\kappa$ B mRNA level was reduced by Nutlin-3, but with ACTD, NF- $\kappa B$ expression was not affected by LPS and/or Nultin-3 (Figure 7A). These data suggest that Nutlin3 blocks LPS-induced NF- $\kappa$ B mRNA level, but does not affect mRNA stability of NF- $\kappa$ B. Furthermore, an NF- $\kappa$ B promoter-driven luciferase reporter was used to measure $\mathrm{NF}-\kappa \mathrm{B}$ transcription, and RPE cells were then treated with LPS and Nutlin-3. Luciferase activity indicated that LPSinduced $N F-\kappa B$ transcription was significantly reduced by Nutlin-3 (Figure 7B). To determine whether Nutlin-3 affects translation or translocation of $\mathrm{NF}-\kappa \mathrm{B}$, translation was inhibited in ARPE-19 cells with CHX, followed by Nutlin-3 and LPS treatment. Western blot analyses of whole cell lysates and nuclear extracts were used to detect NF- $\kappa B$. In whole cell lysates without $\mathrm{CHX}$, LPS-induced NF- $\kappa \mathrm{B}$ expression was reduced by Nutlin-3, but with $\mathrm{CHX}, \mathrm{NF}-\kappa \mathrm{B}$ expression was not affected by LPS and/or Nultin-3 (Figure 7C). In the nuclear extracts, without CHX, LPSinduced NF- $\kappa \mathrm{B}$ translocation was reduced by Nutlin-3, but with $\mathrm{CHX}, \mathrm{NF}-\kappa \mathrm{B}$ translocation was induced with or without Nutlin-3 by LPS (Figure 7D). Similar results were observed in p53 siRNA-transfected ARPE-19 cells (Figure 7, E-H). Taken together, these results suggest that both transcription and translation of NF- $\mathrm{BB}$ are suppressed by MDM2 inhibition independent of p53.

\section{Discussion}

The canonic function of MDM2 in inhibiting cell proliferation and inducing apoptosis through p53 up-regulation is well described in cancer biology. ${ }^{8}$ Our group has described a similar role of MDM2 inhibition in treating pathologic retinal angiogenesis. ${ }^{14}$ Interestingly, MDM2 interacts with nucleic acids and proteins other than $\mathrm{p} 53$ and has numerous functions independent of cell cycle arrest and apoptosis. ${ }^{16-18}$ Recently, a novel p53-independent role of MDM2 was reported in inflammation after postischemic acute kidney injury, where MDM2 increased NF- $\kappa \mathrm{B}$ signaling and MDM2 inhibition reduced NF- $\kappa \mathrm{B}$ and reduced inflammation. ${ }^{11}$ However, it remained unclear if this pathway is relevant in a model of nonvascular inflammation, such as in ocular inflammation. Our work demonstrates that small-molecule MDM2 inhibition robustly inhibits ocular inflammation with or without $\mathrm{p} 53$, both in vitro and in vivo.

Nutlin-3, a small-molecule MDM2 inhibitor, is known to increase p53 levels by binding to MDM2 and disrupting the interaction between MDM2 and p53. ${ }^{19} \mathrm{~A}$ recent study indicates that MDM2 regulates specificity protein $1(\mathrm{Sp} 1)$ and $\mathrm{p} 53$ through a similar binding process but a different proteasomal degradation procedure. ${ }^{20}$ $\mathrm{Sp} 1$ is observed to be down-regulated, independent of 
A

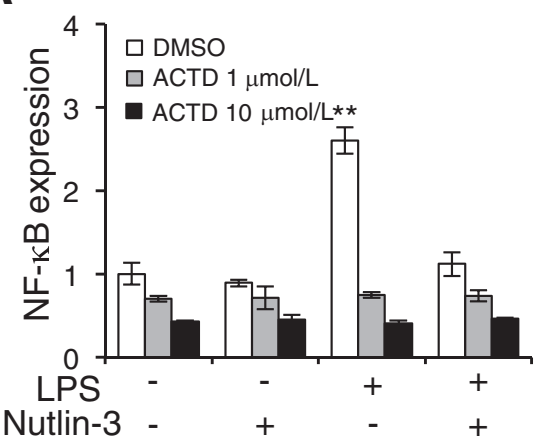

C

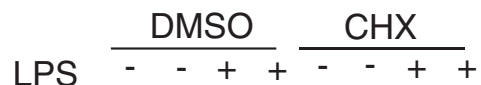

Nutlin-3 - $+-+-+\quad+$

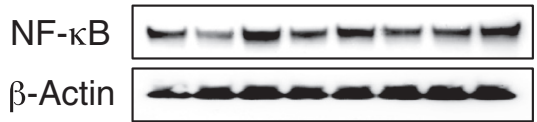

E

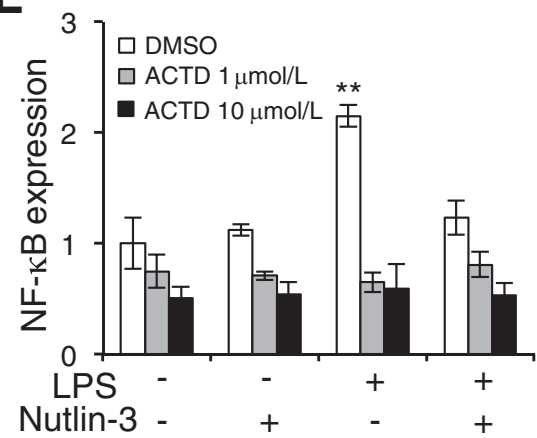

G

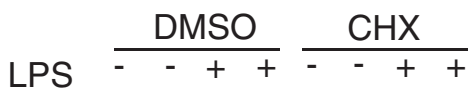

Nutlin-3 - + - + - + +

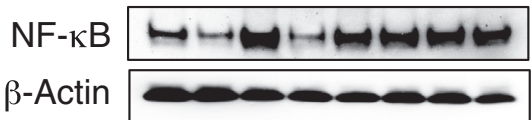

B

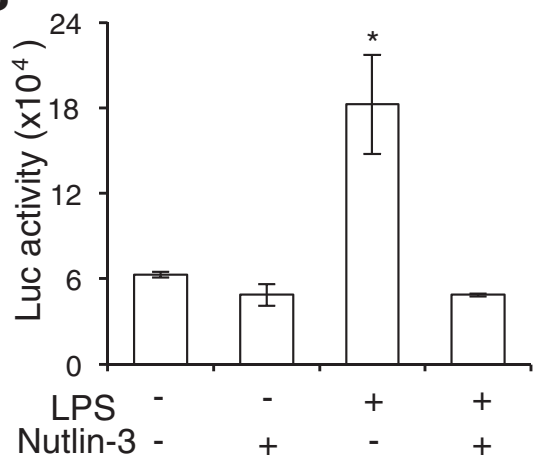

D

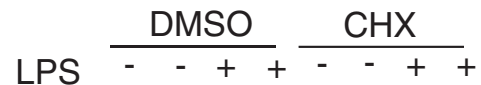

Nutlin-3 - +-+-+-+
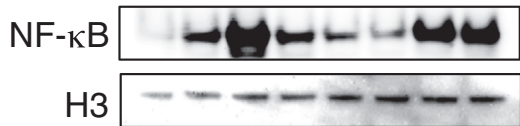

$F$

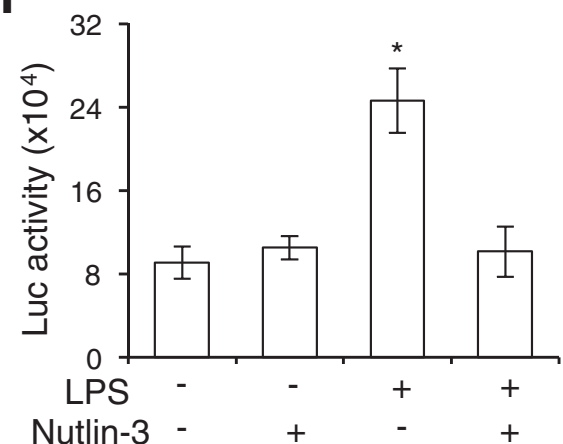

H

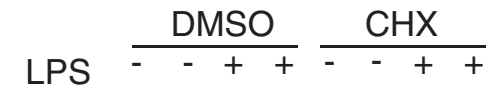

Nutlin-3 - +-+-+-+

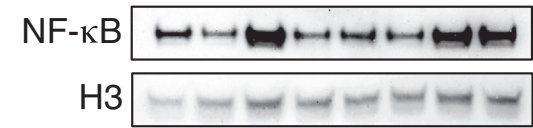

Figure 7 MDM2 inhibition attenuates LPSinduced transcription and translation of NF- $\mathrm{KB}$. A-H: ARPE-19 cells were transfected with control (A-D) or p53 (E-H) siRNA. A and E: Cells were treated with actinomycin D (ACTD) before Nutlin-3 and LPS, and total RNA was used for quantitative PCR analysis of NF-KB. B and F: Cells were transfected with $N F-\kappa B$ promoter-driven luciferase (LuC) reporter and treated with Nutlin-3 and LPS. Cell lysates were used for detecting luciferase activity. Cells were treated with cycloheximide ( $\mathrm{CHX}$ ) before Nutlin-3 and LPS. C, D, G, and H: Whole-cell lysates ( $\mathbf{C}$ and $\mathbf{G}$ ) and nuclear extracts ( $\mathbf{D}$ and H) were processed for Western blot analysis to probe for NF-KB. Data are expressed as means $\pm S D$ of three independent experiments $(\mathbf{A}, \mathbf{B}, \mathbf{E}$, and $\mathbf{F}) .{ }^{*} P<0.05,{ }^{*} P<<0.01$ compared with control. DMS0, dimethyl sulfoxide. p53, by Nutlin through MDM2 inhibition. ${ }^{20}$ Transcription factor $\mathrm{Sp} 1$ and $\mathrm{Sp} 1$-binding sites on $\mathrm{NF}-\kappa \mathrm{B}$ promoter play an important role in transactivating $\mathrm{NF}-\kappa \mathrm{B} .^{21}$ Another study indicates that MDM2 directly binds to Sp1 binding sites on NF- $\mathrm{B}$ promoter and induces NF$\kappa \mathrm{B}$ expression. ${ }^{12}$ In this study, the MDM2 mRNA level was not affected with increasing concentrations of Nutlin-3 (data not shown), so transcriptional inhibition of $N F-\kappa B$ by Nutlin-3 is more likely attributable to Sp1 protein down-regulation other than MDM2 downregulation with Nutlin-3.

We propose Nutlin-3 as a potential therapeutic strategy for the treatment of chronic uveitis. In vitro tests in RPE cells indicated that Nutlin-3 did not inhibit proliferation and cell viability at concentrations $<50 \mu \mathrm{mol} / \mathrm{L}$, which is consistent with other reports. ${ }^{22,23}$ A supratherapeutic dose of Nutlin-3 in vivo did not appear to cause gross ocular toxicity or reduction in retinal nuclear layer thickness. In addition, ocular inflammation or cataract was not observed in Nutlin-3-injected eyes. Moreover, both IVt and i.p. Nutlin-3 effectively inhibited LPS-induced inflammation in both anterior and posterior segments of the eye. Subconjunctival Nutlin-3 injection effectively reversed local LPS-induced inflammatory cell infiltration (data not shown). Extending these observations by using other uveitis models, such as the experimental autoimmune uveitis model, in future studies will be of translational value. Taken together, our work supports further investigation of MDM2 inhibition for the treatment of ocular inflammation. 


\section{Acknowledgments}

We thank Sandra Maansson for processing paraffin sections and assisting with hematoxylin and eosin staining; and Dr. Pankaj Chaudhary for technical assistance.

Y.F. performed and assisted with in vitro and in vivo experiments; W.Z. performed and assisted in all in vivo experiments; A.N. generated the ARPE-19-NF- $\mathrm{B}$ luciferase reporter cells; B.M. repeated the luciferase and cell death assays; all authors contributed to the experimental design; and Y.F. and S.H.C. wrote and edited the manuscript, with the comments and suggestions from all other authors.

\section{Supplemental Data}

Supplemental material for this article can be found at https://doi.org/10.1016/j.ajpath.2018.05.017.

\section{References}

1. Gritz DC, Wong IG: Incidence and prevalence of uveitis in Northern California: the Northern California Epidemiology of Uveitis Study. Ophthalmology 2004, 111:491-500; discussion 500

2. Forrester JV: Uveitis: pathogenesis. Lancet 1991, 338:1498-1501

3. Nussenblatt RB, Gery I: Experimental autoimmune uveitis and its relationship to clinical ocular inflammatory disease. J Autoimmun 1996, 9:575-585

4. Liu D, Ahmet A, Ward L, Krishnamoorthy P, Mandelcorn ED, Leigh R, Brown JP, Cohen A, Kim H: A practical guide to the monitoring and management of the complications of systemic corticosteroid therapy. Allergy Asthma Clin Immunol 2013, 9:30

5. Jobling AI, Augusteyn RC: What causes steroid cataracts? a review of steroid-induced posterior subcapsular cataracts. Clin Exp Optom 2002, 85:61-75

6. Sapir-Pichhadze R, Blumenthal EZ: [Steroid induced glaucoma] Hebrew. Harefuah 2003, 142:137-140. 157

7. Menezo V, Lau C, Comer M, Lightman S: Clinical outcome of chronic immunosuppression in patients with non-infectious uveitis. Clin Exp Ophthalmol 2005, 33:16-21

8. Kawai H, Wiederschain D, Yuan ZM: Critical contribution of the MDM2 acidic domain to p53 ubiquitination. Mol Cell Biol 2003, 23: 4939-4947

9. Tisato V, Voltan R, Gonelli A, Secchiero P, Zauli G: MDM2/X inhibitors under clinical evaluation: perspectives for the management of hematological malignancies and pediatric cancer. J Hematol Oncol 2017, 10:133
10. Burgess A, Chia KM, Haupt S, Thomas D, Haupt Y, Lim E: Clinical overview of MDM2/X-targeted therapies. Front Oncol 2016, $6: 7$

11. Mulay SR, Thomasova D, Ryu M, Anders HJ: MDM2 (murine double minute-2) links inflammation and tubular cell healing during acute kidney injury in mice. Kidney Int 2012, 81:1199-1211

12. Gu L, Findley HW, Zhou M: MDM2 induces NF-kappaB/p65 expression transcriptionally through Sp1-binding sites: a novel, p53independent role of MDM2 in doxorubicin resistance in acute lymphoblastic leukemia. Blood 2002, 99:3367-3375

13. Thomasova D, Mulay SR, Bruns H, Anders HJ: p53-Independent roles of MDM2 in NF-kappaB signaling: implications for cancer therapy, wound healing, and autoimmune diseases. Neoplasia 2012, 14: 1097-1101

14. Chavala SH, Kim Y, Tudisco L, Cicatiello V, Milde T, Kerur N, Claros N, Yanni S, Guaiquil VH, Hauswirth WW, Penn JS, Rafii S, De Falco S, Lee TC, Ambati J: Retinal angiogenesis suppression through small molecule activation of p53. J Clin Invest 2013, 123:4170-4181

15. Sparrow JR, Hicks D, Hamel CP: The retinal pigment epithelium in health and disease. Curr Mol Med 2010, 10:802-823

16. Yang JY, Zong CS, Xia W, Yamaguchi H, Ding Q, Xie X, Lang JY, Lai CC, Chang CJ, Huang WC, Huang H, Kuo HP, Lee DF, Li LY, Lien HC, Cheng X, Chang KJ, Hsiao CD, Tsai FJ, Tsai CH, Sahin AA, Muller WJ, Mills GB, Yu D, Hortobagyi GN, Hung MC: ERK promotes tumorigenesis by inhibiting FOXO3a via MDM2-mediated degradation. Nat Cell Biol 2008, 10:138-148

17. Lundgren K, Montes de Oca Luna R, McNeill YB, Emerick EP, Spencer B, Barfield CR, Lozano G, Rosenberg MP, Finlay CA: Targeted expression of MDM2 uncouples S phase from mitosis and inhibits mammary gland development independent of p53. Genes Dev 1997, 11:714-725

18. Bouska A, Lushnikova T, Plaza S, Eischen CM: Mdm2 promotes genetic instability and transformation independent of p53. Mol Cell Biol 2008, 28:4862-4874

19. Vassilev LT, Vu BT, Graves B, Carvajal D, Podlaski F, Filipovic Z, Kong N, Kammlott U, Lukacs C, Klein C, Fotouhi N, Liu EA: In vivo activation of the p53 pathway by small-molecule antagonists of MDM2. Science 2004, 303:844-848

20. Li H, Zhang Y, Strose A, Tedesco D, Gurova K, Selivanova G: Integrated high-throughput analysis identifies $\mathrm{Sp} 1$ as a crucial determinant of p53-mediated apoptosis. Cell Death Differ 2014, 21: $1493-1502$

21. Hirano F, Tanaka H, Hirano Y, Hiramoto M, Handa H, Makino I, Scheidereit C: Functional interference of Sp1 and NF-kappaB through the same DNA binding site. Mol Cell Biol 1998, 18:1266-1274

22. Bhattacharya S, Ray RM, Chaum E, Johnson DA, Johnson LR: Inhibition of Mdm2 sensitizes human retinal pigment epithelial cells to apoptosis. Invest Ophthalmol Vis Sci 2011, 52:3368-3380

23. Bhattacharya S, Chaum E, Johnson DA, Johnson LR: Age-related susceptibility to apoptosis in human retinal pigment epithelial cells is triggered by disruption of $\mathrm{p} 53-\mathrm{Mdm} 2$ association. Invest Ophthalmol Vis Sci 2012, 53:8350-8366 